\title{
Who Are the People That Experience Soft Usability Problems?
}

\author{
Chajoong Kim and Henri Christiaans \\ School of Industrial Design Engineering \\ Delft University of Technology, The Netherlands \\ $\{$ c.j.kim,h.h.c.m.christiaans\}@tudelft.nl
}

\begin{abstract}
The existing taxonomy of user experience needs to be redefined because of fast technology development in consumer electronic market. Design for all seems prevalent in the field of product design. However, there are ever more doubts about a one-fits-all policy considering the increasing diversity of users and the increasing number of complaints. In our previous studies a taxonomy of 'soft' usability problems related to the use of electronic products has been made. The question posed in this paper is how people behave in the actual use of annoying user-unfriendly products. An experiment was conducted with 33 South Koreans and 23 Americans. A radio alarm clock and a MP3 player were chosen to induce soft usability problems. A questionnaire was used to measure the participants' characteristics. Overall, this study indicates that product operation and user behavior are correlated with specific user characteristics such as age, gender, and culture.
\end{abstract}

Keywords: usability, soft usability problem, user characteristics, culture.

\section{Introduction}

The concept of form of electronic products has been changed a lot over the last decades thanks to the advancement of science and technology. Especially, technology-driven product development has produced electronic products that have all the predictable black-box disadvantages such as the integration of too many functions, operation difficulty and the lack of feedback. The consequence was and still is an increase complaints and product returns; and, disturbing for manufacturers, almost $50 \%$ of these complaints have nothing to do with technical failure [1]. In addition to product complexity, user diversity seems to explain why the product return is increasing [2]. Design for all appears prevalent in the field of product design. However, there are ever more doubts to one-fits-all policy considering the increasing diversity of user [3]. In our previous studies an inventory was made of these so-called 'soft' usability problems based on a survey among people from different countries. The problems could be categorized into six groups: Understanding (difficulties in understanding functions), Performance (low efficiency and compatibility), Sensation (poor sound, touch and temperature quality), Structure (complaints about connections and shape), Maintenance (dissatisfaction with service and cleaning), and Constraint 
(lack of necessary functions, of information/feedback and battery life) [4]. As a follow-up, this study observes the behavior of users when confronted with products that are notorious user-unfriendly and frustrating. Will this behavior be different for different users?

\section{Method}

In order to find out the relationships between user characteristics and soft usability problems, this study first looks into the differences between young and old users, male and female users, and American and South Korean users.

\section{Sample}

The experiment was carried out with 23 American (10 male, 13 female) and 33 South Korean participants (20 male, 13 female), who lived in their home country at the moment they participated in the experiment. They were recruited though an ad. Their age ranged from 20 to 70 .

\section{Instruments}

User characteristics such as demographics, personality, cognitive aspects and consumer complaining behavior, were measured by a questionnaire. To induce soft usability problems in actual product use a radio alarm clock and a mp3 player were selected (Fig. 1). Both electronic products were reported to have many consumer complaints related to soft usability problems.
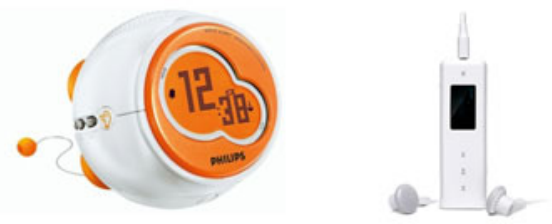

Fig. 1. Radio Alarm Clock and MP3 Player

\section{Procedure}

Participants were individually invited at a location where they felt convenient such as their home, a cafeteria, or a library meeting room. First, the experiment was introduced. This was followed by filling out the first part of the questionnaire. Next, the participant was asked to do several tasks with the radio alarm clock. After this, they filled out the second part of the questionnaire. After, the MP3 player was handed and again several tasks had to be done. Finally, a retrospective interview was taken. All the sessions were videotaped for analysis afterwards.

\section{Results}

Overall, the results indicate that specific user characteristics such as age, gender, and culture are correlated with specific soft usability problems, use behaviors and 
consumer complaining behaviors. Most of the soft usability problems found in our previous studies were identified in both products' operation, but the percentage of soft problems differs (Fig. 2). For instance, Structure is the most frequently heard complain in the radio alarm clock, whereas in the MP3 player it is Understanding.

घadio alarm clock $\square$ MP3 player

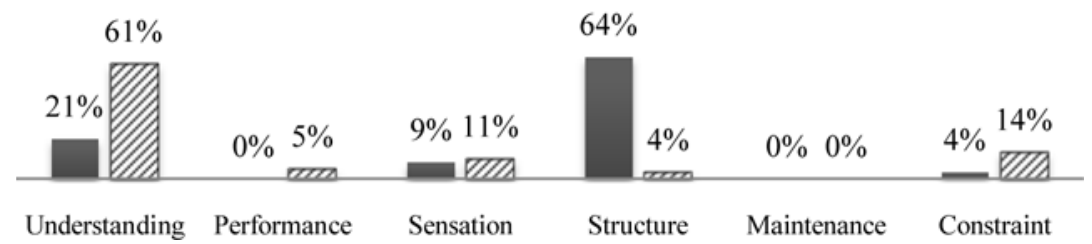

Fig. 2. Soft Usability Problems Comparison between Radio Alarm Clock and MP3 Player

\subsection{Age}

The 20-35 age group can be characterized by seeking for more perfectionism, hardly reading manuals, having better memory, lower uncertainty avoidance, more internal locus of control and better proficiency with electronics compared with the older group (Table 1). The younger group succeeds more in tasks requiring high cognitive load than the older group (Table 2).

Table 1. Group Differences Between 20-35 and 36+ Age Groups

\begin{tabular}{lccccccc}
\hline & \multicolumn{2}{c}{ Age $20-35(\underline{\mathrm{n}}=26)$} & & \multicolumn{2}{c}{ Age $36+(\underline{\mathrm{n}}=30)$} & \\
\cline { 2 - 3 } \cline { 6 - 7 } User characteristics & $\underline{\mathrm{M}}$ & $\underline{\mathrm{SD}}$ & & $\underline{\mathrm{M}}$ & $\underline{\mathrm{SD}}$ & & $\underline{\mathrm{t}}(54)$ \\
\hline Perfectionism & 2.96 & 1.428 & & 2.20 & 1.324 & $2.070^{*}$ \\
Reading manuals & 2.69 & 1.350 & & 3.70 & 1.343 & $-2.794^{* * *}$ \\
Memory & 3.58 & 1.362 & & 2.37 & 1.159 & $3.594^{* * *}$ \\
Uncertainty avoidance & 3.50 & 0.906 & & 4.10 & 1.062 & $-2.256^{*}$ \\
Locus of control & 70.96 & 11.137 & & 77.50 & 10.566 & $-2.252^{*}$ \\
Proficiency with electronics & 2.58 & 0.578 & & 2.07 & 0.785 & $2.733^{* * *}$ \\
\hline
\end{tabular}

$* \mathrm{p}<.01, * * * \mathrm{p}<.001$.

Table 2. Task Success Rate Between 20-35 and 36+ Age Groups

\begin{tabular}{lccc}
\hline Tasks & Age $20-35(\underline{\mathrm{n}}=26)$ & Age $36+(\underline{\mathrm{n}}=30)$ & ${ }^{2}(1)$ \\
\hline Radio alarm clock & 20 & 10 & $8.960^{* * *}$ \\
MP3 player & 19 & 8 & $10.229^{* * *}$ \\
\hline
\end{tabular}

$* * * \mathrm{p}<.001$.

The older group $(\mathrm{M}=4.30, \mathrm{SD}=1.487 ; \mathrm{t}(54)=-4.225, \mathrm{p}=.000)$ is more inclined to complain to the helpdesk than the younger group $(\mathrm{M}=2.54, \mathrm{SD}=1.630)$. There is no significant difference in types of soft problems between the two age groups. 


\subsection{Gender}

Men have higher scores in technical skills and proficiency with electronics but lower scones in Agreeableness (a tendency to be pleasant and accommodating in social situations) than the female group (Table 3). Men have a higher success rate with the radio alarm clock tasks than women (Table 4). They $(\mathrm{M}=1.97, \mathrm{SD}=0.964$; $\mathrm{t}(54)=2.255, \mathrm{p}=.028)$. In spite of the frustrating experience men are also more willing to purchase the radio alarm clock than women $(\mathrm{M}=1.46, \mathrm{SD}=0.706)$.

Table 3. Group Differences Between Male and Female Groups

\begin{tabular}{|c|c|c|c|c|c|}
\hline \multirow[b]{2}{*}{ User characteristics } & \multicolumn{2}{|c|}{ Male $(\underline{n}=30)$} & \multicolumn{2}{|c|}{ Female $(\underline{n}=26)$} & \multirow[b]{2}{*}{$\underline{\mathrm{t}}(54)$} \\
\hline & $\underline{\mathrm{M}}$ & $\underline{\mathrm{SD}}$ & $\underline{\mathrm{M}}$ & $\underline{\mathrm{SD}}$ & \\
\hline Technical skills & $\overline{3.60}$ & $\overline{1.248}$ & $\overline{2.04}$ & $\overline{1.248}$ & $4.668 * * *$ \\
\hline Agreeableness & 29.90 & 3.960 & 34.42 & 4.709 & $-3.905 * * *$ \\
\hline Proficiency with electronics & 2.57 & 0.568 & 2.00 & 0.800 & $3.086 * * *$ \\
\hline
\end{tabular}

Table 4. Task Success Rate Between Male and Female Groups

\begin{tabular}{lccc}
\hline Tasks & Male $(\underline{\mathrm{n}}=30)$ & Female $(\underline{\mathrm{n}}=26)$ & $\chi^{2}(1)$ \\
\hline Radio alarm clock & 29 & 18 & $5.872^{*}$ \\
MP3 player & 17 & 10 & 1.192 \\
\hline
\end{tabular}

$* \mathrm{p}<.01$.

\subsection{Culture}

The South Korean participants complain more about structure (value=8.044, $\mathrm{p}=.005$ ), while the Americans more about understanding (value=6.080, $\mathrm{p}=.014$ ).

The American participants are characterized by higher scores on Openness (respect for all other beings), Agreeableness, Self-efficacy (the belief that one is capable of performing in a certain manner to attain certain goals), but lower scores in Exposure to commercials (Table 5). There are no significant differences in task success rate between both groups. Regarding their reaction on the frustrating experience the American participants are less willing to express their complaints to others $(\mathrm{M}=3.61$, $\mathrm{SD}=1.438 ; \mathrm{t}(54)=2.824, \mathrm{p}=.007)$. In contrast, the South Korean group is more likely to actively express their problems to the helpdesk and give negative comments about the product to their friends $(\mathrm{M}=4.79, \mathrm{SD}=1.269 ; \mathrm{t}(54)=-3.517, \mathrm{p}=.001)$.

Table 5. Group Differences between American and South Korean groups

\begin{tabular}{|c|c|c|c|c|c|}
\hline \multirow[b]{2}{*}{ User characteristics } & \multicolumn{2}{|c|}{ American $(\underline{n}=23)$} & \multicolumn{2}{|c|}{ South Korean $(\mathrm{n}=33)$} & \multirow[b]{2}{*}{$\underline{\mathrm{t}}(54)$} \\
\hline & M & SD & M & SD & \\
\hline Openness & 31.70 & $5 \overline{547}$ & 30.39 & $\overline{4.828}$ & $2.319^{*}$ \\
\hline Agreeableness & 34.30 & 3.936 & 30.39 & 4.828 & $3.209 * * *$ \\
\hline Self-efficacy & 33.30 & 4.343 & 29.91 & 4.179 & $2.944 * * *$ \\
\hline Exposure to commercials & 2.26 & 0.851 & 3.05 & 0.689 & $-3.805 * * *$ \\
\hline
\end{tabular}

$* \mathrm{p}<.01, * * * \mathrm{p}<.001$. 


\section{Conclusions and Discussion}

The main findings in the study are that the type of soft usability problems experienced by users differs between electronic products. Moreover, user behavior such as task success rate, proneness to complaining, and problems experienced are related to specific user characteristics such as age, gender, and culture. They are to some extent consistent with those of our previous studies. How users use electronics and how they complain can partly be predicted when knowing who they are. In this way, this study can provide practical information to the electronic industry and it can lead to an increase in consumer satisfaction.

Acknowledgements. The authors gratefully acknowledge the support of the Innovation-Oriented Research Programme 'Integrated Product Creation and Realization (IOP IPCR)' of the Netherlands Ministry of Economic Affairs.

\section{References}

1. den Ouden, E., Yuan, L., Sonnemans, P.J.M., Brombacher, A.C.: Quality and reliability problems from a consumer's perspective: an increasing problem overlooked by businesses? Quality and Reliability Engineering International 22(7), 821-838 (2006)

2. Brombacher, A.C., Sander, P.C., Sonnemans, P.J.M., Rouvroye, J.L.: Managing product reliability in business processes 'under pressure'. Reliability Engineering \& System Safety 88(2), 137-146 (2005)

3. Abascal, J., Azevedo, L.: Fundamentals of inclusive HCI design. In: Stephanidis, C. (ed.) HCI 2007. LNCS, vol. 4554, pp. 3-9. Springer, Heidelberg (2007)

4. Christiaans, H., Kim, C.: Soft Problems with Consumer Electronics and the Influence of User Characteristics. Paper presented at the 17th International Conference on Engineering Design (ICED 2009), Stanford, USA (2009) 